\title{
Histone deacetylase 5 promotes Wilms' tumor cell proliferation through the upregulation of c-Met
}

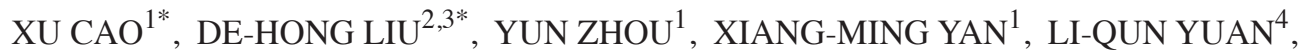 \\ JIAN PAN $^{1}$, MING-CUI FU ${ }^{1}$, TING ZHANG ${ }^{1}$ and JIAN WANG ${ }^{1}$ \\ ${ }^{1}$ Department of Surgery, Childrens' Hospital Affiliated to Soochow University, Soochow University, Suzhou, Jiangsu 215003; \\ ${ }^{2}$ Department of Pediatric Surgery, Ruijin Hospital, Shanghai Jiaotong University School of Medicine, Shanghai 200025; \\ ${ }^{3}$ Department of Pediatric Surgery, Ruijin Hospital North, Shanghai Jiaotong University School of Medicine, Shanghai 201821; \\ ${ }^{4}$ Department of Neurosurgery, The Second Affiliated Hospital of Soochow University, \\ Soochow University, Suzhou, Jiangsu 215004, P.R. China
}

Received October 8, 2014; Accepted June 30, 2015

DOI: $10.3892 / \mathrm{mmr} .2016 .4828$

\begin{abstract}
The histone deacetylase (HDAC) family is comprised of enzymes, which are involved in modulating the majority of critical cellular processes, including transcriptional regulation, apoptosis, proliferation and cell cycle progression. However, the biological function of HDAC5 in Wilms' tumor remains to be fully elucidated. The present study aimed to investigate the expression and function of HDAC5 in Wilm's tumor. It was demonstrated that the mRNA and protein levels of HDAC5 were upregulated in human Wilms' tumor tissues. Overexpression of HDAC5 in G401 cells was observed to significantly promote cellular proliferation, as demonstrated by the results of an MTT assay and bromodeoxyuridine incorporation assay. By contrast, HDAC5 knockdown using small interfering RNA suppressed the proliferation of the G401 cells. At the molecular level, the present study demonstrated that HDAC5 promoted the expression of c-Met, which has been previously identified as an oncogene. In addition, downregulation of c-Met inhibited the proliferative effects of HDAC5 in human Wilms' tumor cells. Taken together, these results suggested that HDAC5 promotes cellular proliferation through the upregulation of c-Met, and may provide a novel therapeutic target for the treatment of patients with Wilms' tumor.
\end{abstract}

Correspondence to: Dr Yun Zhou or Dr Jian Wang, Department of Surgery, Childrens' Hospital Affiliated to Soochow University, Soochow University, 330 Jingde Road, Suzhou, Jiangsu 215003, P.R. China

E-mail: zhangt_zt@126.com

E-mail:wj196312@vip.163.com

*Contributed equally

Key words: Wilms' tumor, histone deacetylase 5, cell proliferation, c-Met

\section{Introduction}

Wilms' tumor (WT), or nephroblastoma, is one of the most prevalent types of solid tumor of the urinary tract in childhood (1). It is estimated that this malignant kidney tumor affects $\sim 1 / 10,000$ children worldwide, which arises from undifferentiated renal precursors and presents with a triphasic histology consisting of stromal, epithelial and blastemal elements $(2,3)$. Although it is treatable with long-term survival rates, the combination of chemo/radiotherapy and surgery can result in severe complications in adulthood $(4,5)$. In addition, the molecular mechanism underlying the pathogenesis of WT remains to be fully elucidated. Therefore, the urgent investigation of novel therapeutic targets for developing anticancer drugs in WT treatment is required.

The histone deacetylase (HDAC) family is comprised of 18 proteins, which are classified into stages I-IV based on their homology and structure (6-8). The epigenetic regulation of gene expression by the HDAC family has been demonstrated to be involved in tumor initiation, progression and metastasis $(9,10)$. Several HDAC antagonists have been observed to inhibit the growth and induce the apoptosis of different types of cancer cell (11-13). In addition, preclinical studies have demonstrated the therapeutic application of HDAC inhibitors as potential anticancer agents (11). HDAC5 belongs to the class II HDAC/acuc/apha family, which is critical in the regulation of cell growth, proliferation, apoptosis and survival (14). A previous study demonstrated that HDAC5 was overexpressed in patients with liver cancer, suggesting that the dysfunction of HDAC5 may be significant in hepatocarcinogenesis (15). Another previous study observed the upregulation of HDAC5 in patients with high-risk medulloblastoma, which was associated with poor survival rates (16). The present study aimed to identify the expression of HDAC5 in WT tissue specimens and investigate the proliferation-promoting function of HDAC5 in human WT cells.

\section{Materials and methods}

Tissue samples. A total of 23 pairs of primary WT tissues (male, 12; female, 11; age, 3-60 months) and normal adjacent tissues 
(male, 13; female, 10; age, 8-64 months) were collected during therapeutic surgery at the Department of Surgery, Childrens' Hospital Affiliated to Soochow University, Soochow University (Suzhou, China). All of the specimens from the WT cases had a size of $1.0 \times 1.0 \times 0.2 \mathrm{~cm}$ and were pathologically diagnosed. Informed consent was obtained from all participants, and the present study was approved by the Institutional Review Board of the Childrens' Hospital Affiliated to Soochow University, Soochow University (Suzhou, China).

Cell culture and transfection. G401 cells were obtained from American Type Culture Collection (Manassas, VA, USA) and were cultured in RPMI 1640 medium (Gibco Life Technologies, Carlsbad, CA, USA), supplemented with $10 \%$ fetal calf serum (Invitrogen Life Technologies), $100 \mathrm{IU} / \mathrm{ml}$ penicillin and $100 \mathrm{mg} / \mathrm{ml}$ streptomycin (both from Sigma-Aldrich, St. Louis, MO, USA). Cells were cultured at $37^{\circ} \mathrm{C}$ in a humidified atmosphere containing $5 \% \mathrm{CO}_{2}$. Plasmids encoding HDAC5-Flag, small interfering RNA (siRNA) specific for HDAC5 (sense 5'-CAUUGCCCACGAGUUCUCACCUGAU-3' and antisense 5'-AUCAGGUGAGAACUCGUGGGCAAUG-3') and siRNA specific for c-Met (sense 5'-AGCCAAUUUAUCA GGAGGUTT-3' and antisense 5'-ACCUCC UGAUAAAUU GGCUTT-3') were purchased from Shanghai GenePharma Co., Ltd. (Shanghai, China). HDAC5 and siRNA c-Met were purchased from Shanghai GenePharma Co., Ltd. (Shanghai, China). The cells were transfected using Lipofectamine 2000 (Invitrogen Life Technologies, Carlsbad, CA, USA), according to the manufacturer's instructions. In brief, cells were seeded into six-well plates and transfected with $30 \mathrm{nM}$ siRNA oligos with $4 \mu 1$ Lipofectamine 2000 at $80 \%$ confluence.

Cell Counting Kit-8 (CCK-8) assay. The cells were seeded into 96-well plates at a density of $6.0 \times 10^{3}$ cells/well. Cell viability was assessed using a CCK-8 assay (Beyotime Institute of Biotechnology, Haimen, China). The absorbance of each well was read using a spectrophotometer (4015-000; Thermo Fisher Scientific, Waltham, MA, USA) at $450 \mathrm{~nm}$.

Bromodeoxyuridine (BrdU) incorporation analysis. BrdU incorporation assay was performed using a BrdU Cell Proliferation kit (Roche Diagnostics, Indianapolis, IN, USA) to analyze cell growth. Briefly, the cells were incubated with BrdU for $6 \mathrm{~h}$, rinsed and then incubated with a fluorescein isothiocyanate-labeled antibody against $\mathrm{BrdU}$ for $30 \mathrm{~min}$. The stained cells were then analyzed using a fluorescence microplate reader (2350; EMD Millipore, Billerica, MA, USA). Fold changes in BrdU incorporation were normalized against the mean fluorescence intensity of the control group.

Reverse transcription-quantitative polymerase chain reaction $(R T-q P C R)$. Frozen tissues or cells were homogenized using TRIzol reagent (Invitrogen Life Technologies) and total RNA was isolated using a Qiagen RNeasy Lipid Tissue Mini kit (Qiagen, Hilden, Germany) according to the manufacturer's instructions. Reverse transcription was performed using a Takara RNA PCR kit (Takara Bio, Inc., Otsu, Japan) according to the manufacturer's instructions. RT-qPCR was performed using SYBR Green Premix Ex Taq (Takara Bio, Inc.) on an ABI 7500 Cycling system (Applied Biosystems,
$\mathbf{A}$

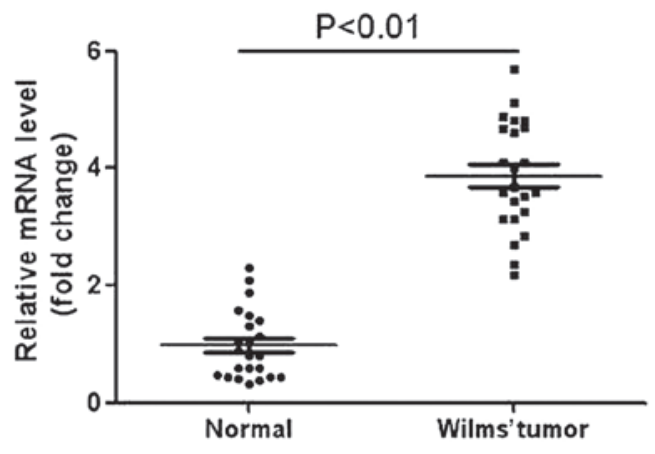

B

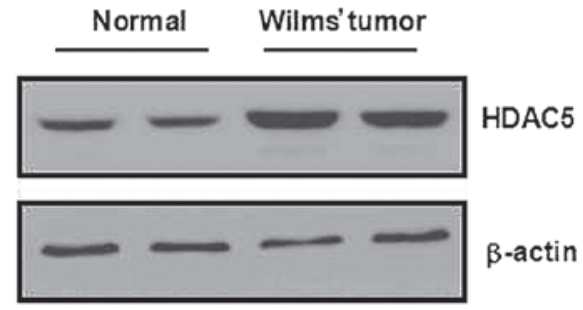

Figure 1. Upregulation of HDAC5 in Wilms' tumor tissues. The mRNA and protein levels of HDAC5 were determined using (A) reverse transcription-quantitative polymerase chain reaction and (B) western blot analysis, respectively, in human Wilms' tumor tissues and normal tissues. Data are presented as the mean \pm standard deviation. HDAC5, histone deacetylase 5 .

Life Technologies, Thermo Fisher Scientific) to determine the expression levels of the genes of interest. The cycling parameters were as follows: Initial denaturation at $95^{\circ} \mathrm{C}$ for $30 \mathrm{sec}$, followed by a two-step program of $95^{\circ} \mathrm{C}$ for $5 \mathrm{sec}$ and $60^{\circ} \mathrm{C}$ for $31 \mathrm{sec}$ over 40 cycles. Experiments were performed in triplicate. The primers were obtained from Invitrogen Life Technologies and the sequences were as follows: HDAC5 forward, 5'-CTCAAGCAGCAGCAGCAGCTCCA-3' and reverse, 5'-CCTTCTGTTTAAGCCTCGAACG-3'; c-Met forward, 5'- CCTTCGAAAGCAACCATTTTACG-3' and reverse, 5'-TTACTGACATACGCGGCTTGCGC-3'. The expression levels were quantified using the $2^{-\Delta \Delta C \mathrm{Ct}}$ method.

Western blot analysis. Total protein was extracted with radioimmunoprecipitation assay lysis buffer (Beyotime Institute of Biotechnology) and quantified using the bicinchoninic acid assay (BAC kit; Beyotime Institute of Biotechnology). The proteins $(20 \mu \mathrm{g})$ were fractionated by $10 \%$ SDS-PAGE and transferred to polyvinylidene difluoride membranes (EMD Millipore). The membrane was blocked in $4 \%$ dried milk at room temperature for $1 \mathrm{~h}$ and incubated with primary antibodies at $4^{\circ} \mathrm{C}$ overnight. Rabbit anti-HDAC5 monoclonal antibody (cat no. 3443S; 1:1,000; Cell Signaling Technology, Inc., Beverly, MA, USA) and mouse anti-c-Met monoclonal antibody (cat no. sc-162; 1:1,000; Santa Cruz Biotechnology, Inc., Santa Cruz, CA, USA) were used as primary antibodies. Subsequently, the membrane was incubated with horseradish peroxidase-conjugated secondary antibodies (mouse-IgG; 1:5,000; Beyotime Institute of Biotechnology, Shanghai, China) for $1 \mathrm{~h}$ at room temperature and detected with an enhanced chemiluminescence system (Roche Diagnostics), according to the manufacturer's instructions. $\beta$-actin was purchased from Santa Cruz Biotechnology, Inc., which was used as a loading control. 
$\mathbf{A}$

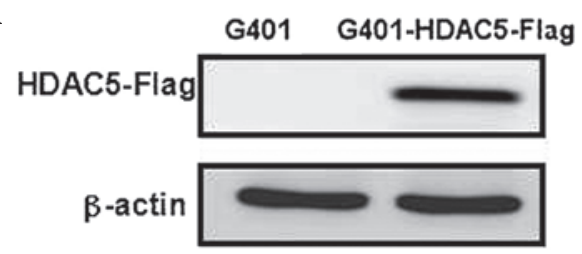

B

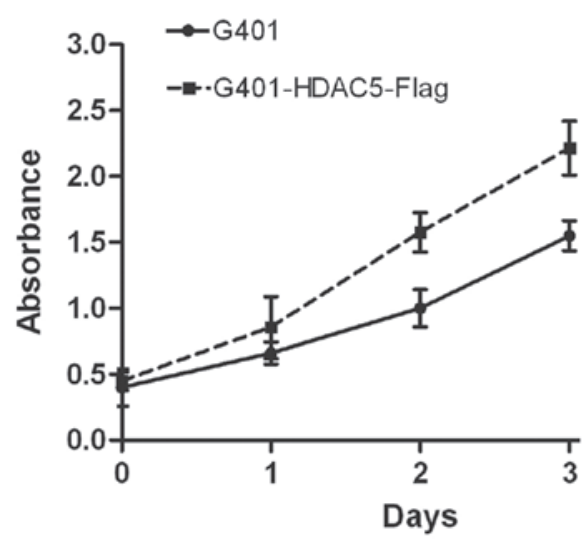

C

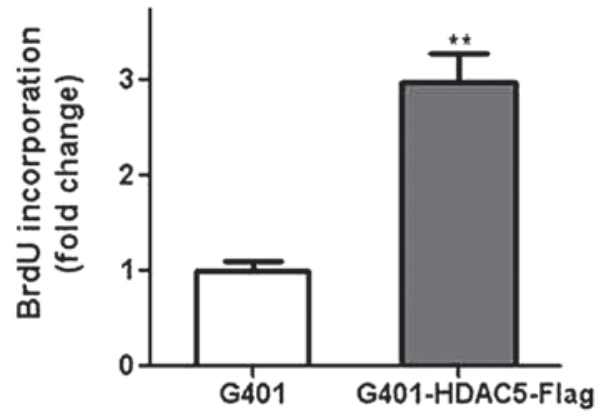

Figure 2. Overexpression of HDAC5 promotes cell growth in Wilms' tumor. (A) Protein expression of HDAC5 was determined using western blot analysis in G401 cells transfected with plasmids encoding HDAC5-Flag. Following transfection, the proliferation of G401 cancer cells was measured using (B) Cell Counting Kit- 8 and (C) BrdU incorporation assays. ${ }^{* *} \mathrm{P}<0.01$, compared with the G401 group. Data are expressed as the mean \pm standard deviation. HDAC5, histone deacetylase 5; BrdU, bromodeoxyuridine.

Statistical analysis. Values are expressed as the mean \pm standard deviation, based on three separate experiments. One-way analysis of variance was used for comparison between multiple groups and Student's t-test was used for comparison between two groups. $\mathrm{P}<0.05$ was considered to indicate a statistically significant difference. All statistical analyses were performed using SPSS, version 13.0 (SPSS, Inc., Chicago, IL, USA).

\section{Results}

Upregulation of HDAC5 in WT tissues. The mRNA expression levels of HDAC5 were examined in 23 paired WT and adjacent non-tumor tissues using RT-qPCR. It was observed that the expression of HDAC5 was significantly increased in the tumor tissues (Fig. 1A). In addition, the protein expression level of HDAC5 was determined using western blot analysis, which revealed increased expression levels of HDAC5 in the WT samples, compared with the adjacent normal samples (Fig. 1B). These data suggested that HDAC5 may be important in WT pathogenesis.
A

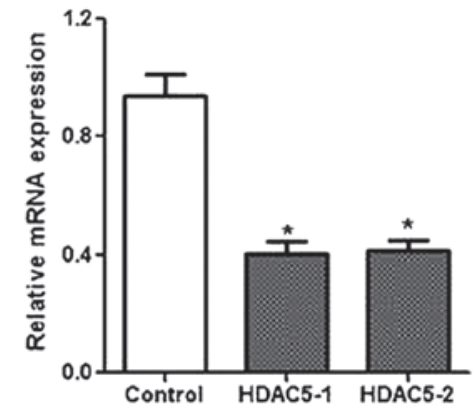

B
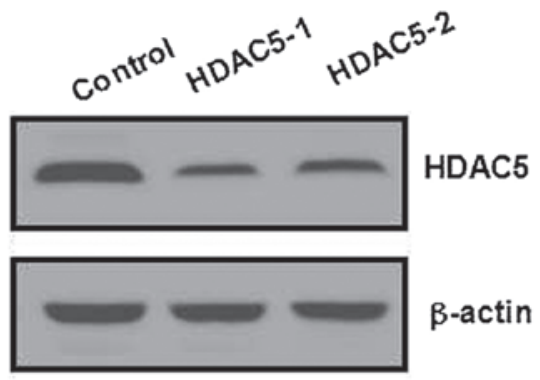

C

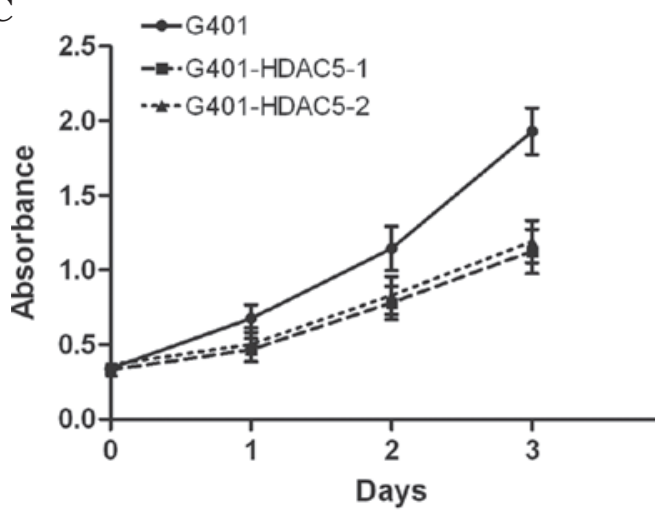

D

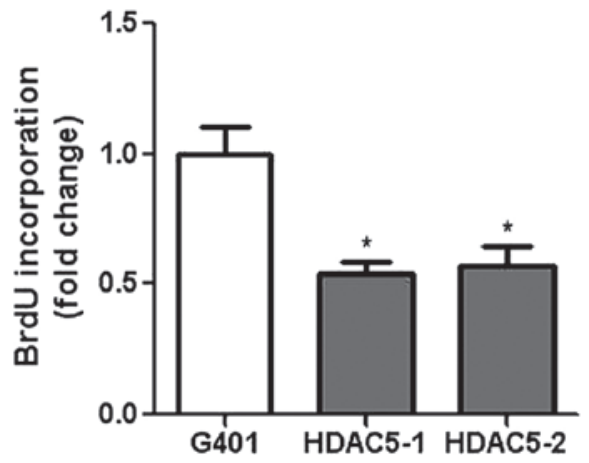

Figure 3. Downregulation of HDAC5 suppresses the proliferation of Wilms' tumor cells. HDAC5 knockdown was confirmed using (A) reverse transcription-quantitative polymerase chain reaction and (B) western blot analysis in G401 cells transfected with siRNA oligos targeting HDAC5. The cell proliferation was then measured using (C) Cell Counting Kit- 8 and (D) BrdU incorporation assays in G401 cells transfected with HDAC5 siRNA oligos. ${ }^{*} \mathrm{P}<0.05$, compared with the G401 group. Data are expressed as the mean \pm standard deviation. HDAC5, histone deacetylase 5; BrdU, bromodeoxyuridine.

HDAC5 promotes cellular proliferation of WT. In order to elucidate the biological role of HDAC5 in WT, G401 cells were transfected with a recombinant plasmid containing HDAC5 
A

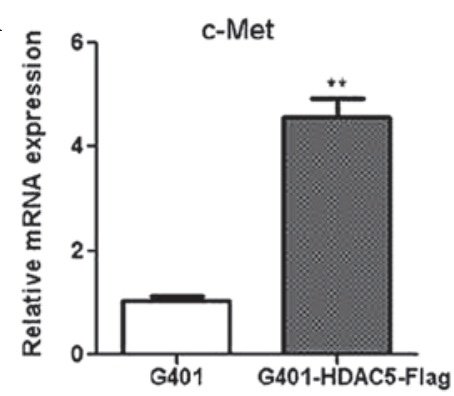

D

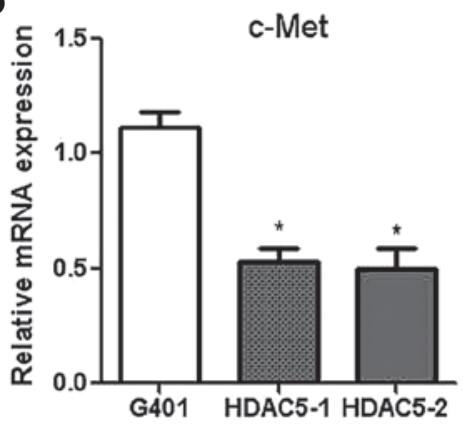

B

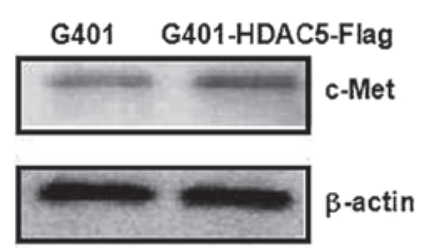

E

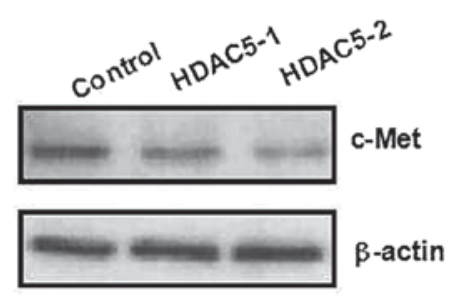

C

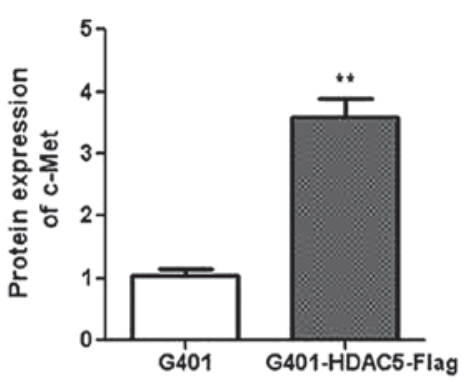

F

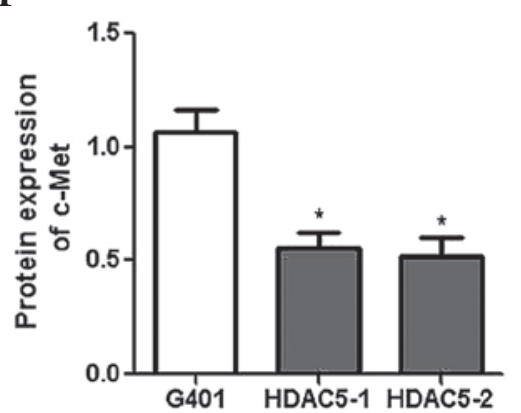

Figure 4. HDAC5 promotes the expression of c-Met in G401 cells. The expression level of c-Met in G401 cells overexpressing HDAC5 was examined using (A) RT-qPCR and (B and C) western blot analysis. Following transfection with siRNA oligos targeting HDAC5, the expression of c-Met was determined using (D) RT-qPCR and (E and F) western blot analysis in G401 cells. Relative band intensities of each protein were quantified using densitometry. ${ }^{*} \mathrm{P}<0.05$ and ${ }^{* *} \mathrm{P}<0.01$, compared with the G401 group. HDAC5, histone deacetylase 5; RT-qPCR, reverse transcription-quantitative polymerase chain reaction.

A

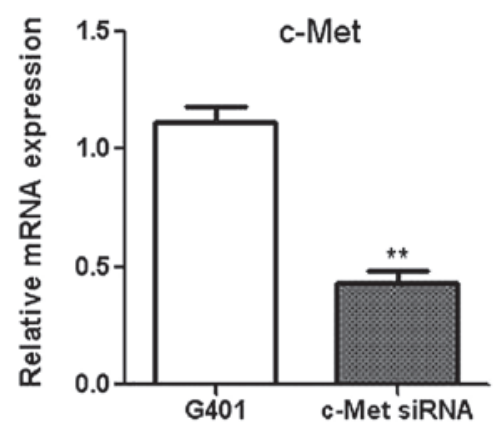

C

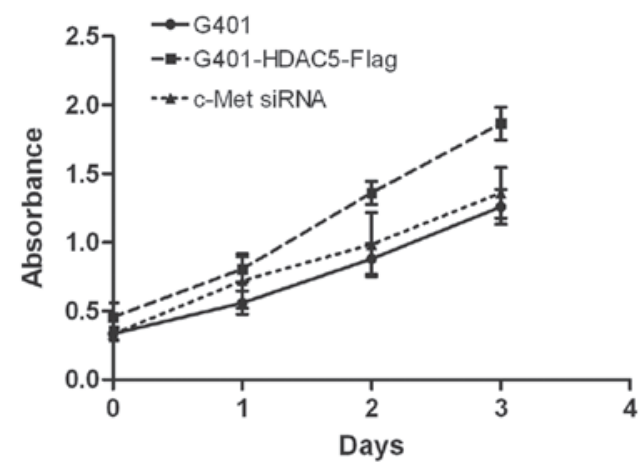

B

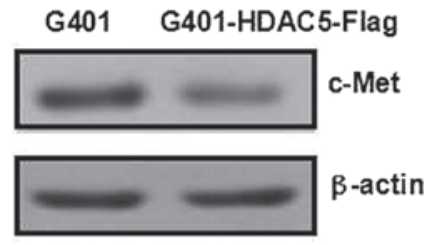

D

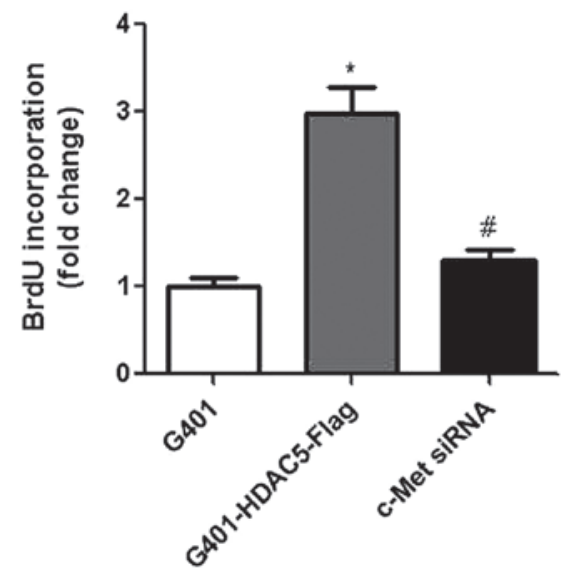

Figure 5. HDAC5 promotes cell proliferation through the regulation of c-Met. Downregulation of c-Met was confirmed using (A) reverse transcription-quantitative polymerase chain reaction and (B) western blot analysis in the G401 cells transfected with c-Met siRNA oligos. (C) Cell Counting Kit- 8 and (D) BrdU incorporation assays were performed to measure cell proliferation in the G401 cells overexpressing HDAC5, with or without c-Met siRNA. ${ }^{*} \mathrm{P}<0.05$, ${ }^{* *} \mathrm{P}<0.01$ and " $\mathrm{P}<0.05$, compared with G401 cells overexpressing HDAC5. HDAC5, histone deacetylase 5; BrdU, bromodeoxyuridine; siRNA, small interfering RNA.

(Fig. 2A). The subsequent CCK-8 assay demonstrated that overexpression of HDAC5 clearly promoted the proliferation of the WT cells $(\mathrm{P}<0.05)$ (Fig. 2B). BrdU incorporation is a standard method to measure DNA synthesis and is considered to be a surrogate procedure for the evaluation of proliferation (17). In the present study, the G401 cells overexpressing 
HDAC5 exhibited a faster growth rate, compared with the control G401 cells (Fig. 2C), consistent with the results of the CCK-8 assay. In addition, the G401 cells were transfected with siRNA targeting HDAC5, and the subsequent downregulation of the target gene was confirmed using RT-qPCR and western blotting (Fig. 3A and B). The data indicated that the downregulation of HDAC5 significantly decreased the rate of proliferation of the G401 cells (Fig. 3C and D). Taken together, these results indicated that HDAC5 may be a positive regulator of cellular proliferation in WT.

HDAC5 regulates WT growth through $c$-Met. The present study also investigated the molecular mechanism underlying the proliferative effect of HDAC5, and the results demonstrated that the mRNA expression of c-Met was increased by $>4$-fold in the G401 cells overexpressing HDAC5, compared with the control G401 cells (Fig. 4A). Western blot analysis also confirmed the upregulation of c-Met in the HDAC5-transfected cells (Fig. 4B and C). By contrast, the expression of c-Met was reduced in the G401 cells following the downregulation of HDAC5 by siRNA interference (Fig. 4D-F). Taken together, these data suggested that HDAC5 was an upstream regulator of c-Met. In order to ascertain whether the induction of c-Met is a prerequisite for the proliferative function of HDAC5, the expression of c-Met was suppressed using siRNA oligos (Fig. 5A and B). Notably, the downregulation of c-Met inhibited the proliferative effects of HDAC5 on the G401 cells (Fig. 5C and D), suggesting that the proliferation-promoting function of HDAC5 was involved in the regulation of c-Met.

\section{Discussion}

Accumulating evidence has demonstrated that several members of the HDAC family are critical in promoting carcinogenesis (18). However, the biological function of HDAC5 in WT remains to be fully elucidated. In the present study, the expression levels of HDAC5 were examined in WT specimens and adjacent normal tissue specimens, and the proliferative effect of HDAC5 was investigated in human WT cells.

HDACs have been reported to remove the acetyl groups from the $\mathrm{N}$-acetyl-sites on the histone, thus modifying the chromatin structure and modulating the expression levels of several genes (19). The aberrant expression levels of HDAC family members have been associated with tumor initiation and progression (18). HDAC5 belongs to the class II HDAC family and is a critical regulator in cellular proliferation, cell cycle progression and apoptosis in several cancer cell lines and animal models $(20,21)$. A previous study demonstrated that HDAC5 is significantly overexpressed in high-risk medulloblastoma, compared with low-risk medulloblastoma, and its expression is associated with poor survival rates, suggesting that HDAC5 may be an important marker for risk stratification (18). Another study demonstrated that HDAC5 promotes the progression of osteosarcoma through upregulation of the twist 1 oncogene (22). In the present study, it was demonstrated that HDAC5 was significantly increased in human WT samples, compared with adjacent normal tissues, suggesting that HDAC5 may be critical in the pathogenesis of WT. In addition, in vitro investigation demonstrated that overexpression of
HDAC5 in G401 cells significantly increased the level of cell proliferation, compared with normal G401 cells. By contrast, HDAC5 knockdown using siRNA reduced cell growth, suggesting that HDAC5 acts as a positive regulator in the proliferation of WT cells.

The cell surface receptor tyrosine kinase, c-Met, is overexpressed in a several types of malignancy, including hepatocellular carcinoma (23), lung cancer (24) and ovarian carcinoma (25). c-Met is important in cellular proliferation, migration and metastasis (26). A number of studies have demonstrated that increased c-Met signaling contributes to carcinogenesis through several pathways, including the focal adhesion kinase, phosphatidyl inositol 3-kinase and extracellular signal-regulated kinase pathways (27-29). It has been demonstrated that the downregulation of c-Met produces antitumor effects, predominantly based on anti-proliferation and anti-angiogenesis, in several types of cancer cell (30-32). In the present study, the upregulation of HDAC5 was observed to promote the mRNA and protein expression levels of c-Met in G401 cells. In addition, c-Met depletion significantly reversed the proliferation-promoting function of HDAC5 in human WT cells.

In conclusion, the present study demonstrated that HDAC5 promoted the proliferation of WT through upregulation of c-Met. These findings provide further insight into the pathogenic mechanisms of WT and suggests HDAC5 as a potential therapeutic target.

\section{Acknowledgements}

The present study was supported by grants from the Science Foundation of Jiangsu Province Health Department (grant nos. Q201304 and Q201503), the Natural Science Foundation of Jiangsu Province of China (grant nos. BK2011312 and BL2012051) and the Natural Science Foundation of China (no. 81502496).

\section{References}

1. Turnbull C, Perdeaux ER, Pernet D, Naranjo A, Renwick A, Seal S, Munoz-Xicola RM, Hanks S, Slade I, Zachariou A, et al: A genome-wide association study identifies susceptibility loci for Wilms tumor. Nat Genet 44: 681-684, 2012.

2. Wegert J, Bausenwein S, Kneitz S, Roth S, Graf N, Geissinger E and Gessler M: Retinoic acid pathway activity in Wilms tumors and characterization of biological responses in vitro. Mol Cancer 10: 136, 2011.

3. Tian F, Yourek G, Shi X and Yang Y: The development of Wilms tumor: From WT1 and microRNA to animal models. Biochim Biophys Acta 1846: 180-187, 2014.

4. Sonn G and Shortliffe LM: Management of Wilms tumor: Current standard of care. Nat Clin Pract Urol 5: 551-560, 2008.

5. Acipayam C, Sezgin G, Bayram İ, Y1lmaz S, Özkan A, Tuncel DA, Tanyeli A and Küpeli S: Treatment of Wilms tumor using carboplatin compared to therapy without carboplatin. Pediatr Blood Cancer 61: 1578-1583, 2014.

6. West AC, Mattarollo SR, Shortt J, Cluse LA, Christiansen AJ, Smyth MJ and Johnstone RW: An intact immune system is required for the anticancer activities of histone deacetylase inhibitors. Cancer Res 73: 7265-7276, 2013.

7. Wagner JM, Hackanson B, Lübbert M and Jung M: Histone deacetylase (HDAC) inhibitors in recent clinical trials for cancer therapy. Clin Epigenetics 1: 117-136, 2010.

8. Witt O, Deubzer HE, Milde T and Oehme I: HDAC family: What are the cancer relevant targets? Cancer Lett 277: 8-21, 2009.

9. Song SH, Han SW and Bang YJ: Epigenetic-based therapies in cancer: Progress to date. Drugs 71: 2391-2403, 2011. 
10. Vigushin DM and Coombes RC: Histone deacetylase inhibitors in cancer treatment. Anticancer Drugs 13: 1-13, 2002.

11. Khan $\mathrm{O}$ and La Thangue NB: HDAC inhibitors in cancer biology: Emerging mechanisms and clinical applications. Immunol Cell Biol 90: 85-94, 2012.

12. Cao B, Li J, Zhu J, Shen M, Han K, Zhang Z, Yu Y, Wang Y, Wu D, Chen S, et al: The antiparasitic clioquinol induces apoptosis in leukemia and myeloma cells by inhibiting histone deacetylase activity. J Biol Chem 288: 34181-34189, 2013.

13. Lai F, Jin L, Gallagher S, Mijatov B, Zhang XD and Hersey P: Histone deacetylases (HDACs) as mediators of resistance to apoptosis in melanoma and as targets for combination therapy with selective BRAF inhibitors. Adv Pharmacol 65: 27-43, 2012

14. Zhang Y, Matkovich SJ, Duan X, Diwan A, Kang MY and Dorn GN II: Receptor-independent protein kinase $\mathrm{C}$ alpha (PKCalpha) signaling by calpain-generated free catalytic domains induces HDAC5 nuclear export and regulates cardiac transcription. J Biol Chem 286: 26943-26951, 2011.

15. Feng GW, Dong LD, Shang WJ, Pang XL, Li JF, Liu L and Wang Y: HDAC5 promotes cell proliferation in human hepatocellular carcinoma by up-regulating Six1 expression. Eur Rey Med Pharmacol Sci 18: 811-816, 2014.

16. Milde T, Oehme I, Korshunov A, Kopp-Schneider A, Remke M, Northcott P, Deubzer HE, Lodrini M, Taylor MD, von Deimling A, et al: HDAC5 and HDAC9 in medulloblastoma: Novel markers for risk stratification and role in tumor cell growth. Clin Cancer Res 16: 3240-3252, 2010.

17. Actis M, Inoue A, Evison B, Perry S, Punchihewa C and Fujii N: Small molecule inhibitors of PCNA/PIP-box interaction suppress translesion DNA synthesis. Bioorg Med Chem 21: 1972-1977, 2013.

18. Li Z and Zhu WG: Targeting histone deacetylases for cancer therapy: From molecular mechanisms to clinical implications Int J Biol Sci 10: 757-770, 2014.

19. Rincon-Arano H, Halow J, Delrow JJ, Parkhurst SM and Groudine M: UpSET recruits HDAC complexes and restricts chromatin accessibility and acetylation at promoter regions. Cell 151: 1214-1228, 2012.

20. Marek L, Hamacher A, Hansen FK, Kuna K, Gohlke H, Kassack MU and Kurz T: Histone deacetylase (HDAC) inhibitors with a novel connecting unit linker region reveal a selectivity profile for HDAC4 and HDAC5 with improved activity against chemoresistant cancer cells. J Med Chem 56: 427-436, 2013.

21. Peixoto P, Castronovo V, Matheus N, Polese C, Peulen O, Gonzalez A, Boxus M, Verdin E, Thiry M, Dequiedt F and Mottet D: HDAC5 is required for maintenance of pericentric heterochromatin and controls cell-cycle progression and survival of human cancer cells. Cell Death Differ 19: 1239-1252, 2012.
22. Chen J, Xia J, Yu YL, Wang SQ, Wei YB, Chen FY, Huang GY and Shi JS: HDAC5 promotes osteosarcoma progression by upregulation of Twist 1 expression. Tumour Biol 35: 1383-1387, 2014.

23. Kondo S, Ojima H, Tsuda H, Hashimoto J, Morizane C, Ikeda M, Ueno H, Tamura K, Shimada K, Kanai Y and Okusaka T: Clinical impact of c-Met expression and its gene amplification in hepatocellular carcinoma. Int J Clin Oncol 18: 207-213, 2013

24. Lee JM, Yoo JK, Yoo H, Jung HY, Lee DR, Jeong HC, Oh SH, Chung HM and Kim JK: The novel miR-7515 decreases the proliferation and migration of human lung cancer cells by targeting c-Met. Mol Cancer Res 11: 43-53, 2013.

25. Koon EC, Ma PC, Salgia R, Christensen JG, Berkowitz RS and Mok SC: Effect of a c-Met-specific, ATP-competitive small-molecule inhibitor SU11274 on human ovarian carcinoma cell growth, motility and invasion. Int J Gynecol Cancer 18: 976-984, 2008

26. Goetsch L, Caussanel V and Corvaia N: Biological significance and targeting of c-Met tyrosine kinase receptor in cancer. Front Biosci (Landmark Ed) 18: 454-473, 2013.

27. Lee YY, Kim HP, Kang MJ, Cho BK, Han SW, Kim TY and Yi EC: Phosphoproteomic analysis identifies activated MET-axis $\mathrm{PI} 3 \mathrm{~K} / \mathrm{AKT}$ and MAPK/ERK in lapatinib-resistant cancer cell line. Exp Mol Med 45: e64, 2013.

28. Nam HJ, Chae S, Jang SH, Cho H and Lee JH: The PI3K-Akt mediates oncogenic Met-induced centrosome amplification and chromosome instability. Carcinogenesis 31: 1531-1540, 2010.

29. Guessous F, Yang Y, Johnson E, Marcinkiewicz L, Smith M, Zhang Y, Kofman A, Schiff D, Christensen J and Abounader R: Cooperation between c-Met and focal adhesion kinase family members in medulloblastoma and implications for therapy. Mol Cancer Ther 11: 288-297, 2012.

30. Hong SW, Jung KH, Park BH, Zheng HM, Lee HS, Choi MJ, Yun JI, Kang NS, Lee J and Hong SS: KRC-408, a novel c-Met inhibitor, suppresses cell proliferation and angiogenesis of gastric cancer. Cancer Lett 332: 74-82, 2013.

31. Yang X, Yang Y, Tang S, Tang H, Yang G, Xu Q and Wu J: Anti-tumor effect of polysaccharides from Scutellaria barbata D. Don on the 95-D xenograft model via inhibition of the C-met pathway. J Pharmacol Sci 125: 255-263, 2014.

32. Zhang HT, Wang L, Ai J, Chen Y, He CX, Ji YC, Huang M, Yang JY, Zhang A, Ding J and Geng MY: SOMG-833, a novel selective c-MET inhibitor, blocks c-MET-dependent neoplastic effects and exerts antitumor activity. J Pharmacol Exp Ther 350: 36-45, 2014. 